\title{
Microscopic model of the THz field enhancement in a metal nanoslit
}

\author{
Novitsky, Andrey; Zalkovskij, Maksim; Malureanu, Radu; Lavrinenko, Andrei
}

Published in:

Optics Communications

Link to article, DOI:

10.1016/j.optcom.2011.08.019

Publication date:

2011

Document Version

Early version, also known as pre-print

Link back to DTU Orbit

Citation (APA):

Novitsky, A., Zalkovskij, M., Malureanu, R., \& Lavrinenko, A. (2011). Microscopic model of the THz field enhancement in a metal nanoslit. Optics Communications, 284(23), 5495.

https://doi.org/10.1016/j.optcom.2011.08.019

\section{General rights}

Copyright and moral rights for the publications made accessible in the public portal are retained by the authors and/or other copyright owners and it is a condition of accessing publications that users recognise and abide by the legal requirements associated with these rights.

- Users may download and print one copy of any publication from the public portal for the purpose of private study or research.

- You may not further distribute the material or use it for any profit-making activity or commercial gain

- You may freely distribute the URL identifying the publication in the public portal 


\title{
Microscopic model of the $\mathrm{THz}$ field enhancement in a metal nanoslit
}

\author{
A. Novitsky, M. Zalkovskij, R. Malureanu, and A. Lavrinenko \\ DTU Fotonik, Department of Photonics Engineering, Technical University of Denmark, \\ Ørsteds plads 343, DK-2800 Kgs. Lyngby, Denmark; anov@fotonik.dtu.dk
}

\begin{abstract}
We discuss the strong THz-field enhancement effect in a metal slit of dozens of nanometers sizes reported recently. Proposed simple microscopic model considers electric charges induced at the edges of the slit by a polarized incident wave. These charges contribute then to the field in the slit. The model is capable of explaining peculiarities of the field enhancement phenomenon such as an inverse frequency dependence of the enhancement factor. It provides closed-form expressions for the enhancement factor and field mapping inside the slit having only one fitting parameter. The model predicts influence of the slit shape on the field enhancement.
\end{abstract}

Keywords: metallic nanoslit; $\mathrm{THz}$ radiation; field enhancement

\section{Introduction}

Recently the strong field enhancement of a $\mathrm{THz}$ wave impinging on a nanoslit made in a thin (in comparison with the skin depth) metal slab has been reported [1]. The field in the slit is appeared to be much stronger (by up to a thousand times) than the incident one. It is important that the enhancement is achieved for a single slit in a broad spectral range providing non-resonant behavior of the structure. Therefore, the slit strongly focuses the electromagnetic radiation acting as an optical antenna [2]. The field enhancement strongly depends on the slit width and inversely proportional to the frequency of the incident $\mathrm{THz}$ wave. That is why the field enhancement is greater for the lower frequencies. Note that the conditions of appearing of the field enhancement effect mentioned above differ from those needed for the extraordinary transmission [3], the fact which is also mentioned in the recent 
review [4]. Thus, we consider the enhancement of $\mathrm{THz}$ fields in a nanoscaled slit to be a distinct phenomenon requiring a simple physical model for better insight and accurate quantitative description.

The field enhancement has been proved experimentally and numerically. For estimation of the enhancement the correlation between the near and the far field (what comes to a detector) according to the diffraction theory is used $[1,5]$. The experimental results obtained are entirely confirmed by the full-vectorial simulations $[1,6]$. The theory of the interaction of light with metal apertures was first considered by Bethe [7] and then complemented by Bouwkamp [8]. The field inside an rectangular aperture can be found using Kirchhoff's diffraction integral or Reileigh's wave expansion of both transmitted and reflected waves. In the latter case, the field inside the slit is written by means of waveguide's modes expansion. Then the connection of the fields at the boundaries results in the closed-form solution for the field inside the slit $[5,2]$. Another model of the electric field enhancement, so-called the local capacitor model, was introduced by Kang et al [9]. It is based on the idea of the " $\lambda$-zone" capacitance and provides the simple description of the lightning rod effect in nanoplasmonics (the field gain near sharp edges). The authors obtained full agreement with rigorous calculations. The local capacitor model is able to predict the value of enhancement for different shapes of the slit, if the capacitance is known. The parametric model presented in the current paper is simpler than the model in Ref. [9] and provides both enhancement and the field distribution. We also emphasize that our model is founded on the Drude-Lorentz model of the electrons in the metal film, what provides us with qualitative understanding of how the dynamics of the electrons causes the field enhancement.

The electric field enhancement by a single metal nanoslit in the subskin-depth regime can be applied in plasmonics, light focusing and beaming $[2,11,10,12]$. At the same time the exciting future applications involving nonliner materials are expected. Due to the strong field enhancement the nonlinearity may cause significant changes in, e.g., refractive index, what can be used in designing new types of metamaterial modulators, waveguides and photonic circuits $[13,14,15,16]$.

The paper is organized as follows. In section 2 the microscopic model is discussed. The model is grounded on the Drude-Lorentz theory of a classical electron and electrostatic approximation. In section 3 we confirm the correctness of the model by comparing with numerical data and experiment known from the literature [1]. The phenomenological parameter of the model 


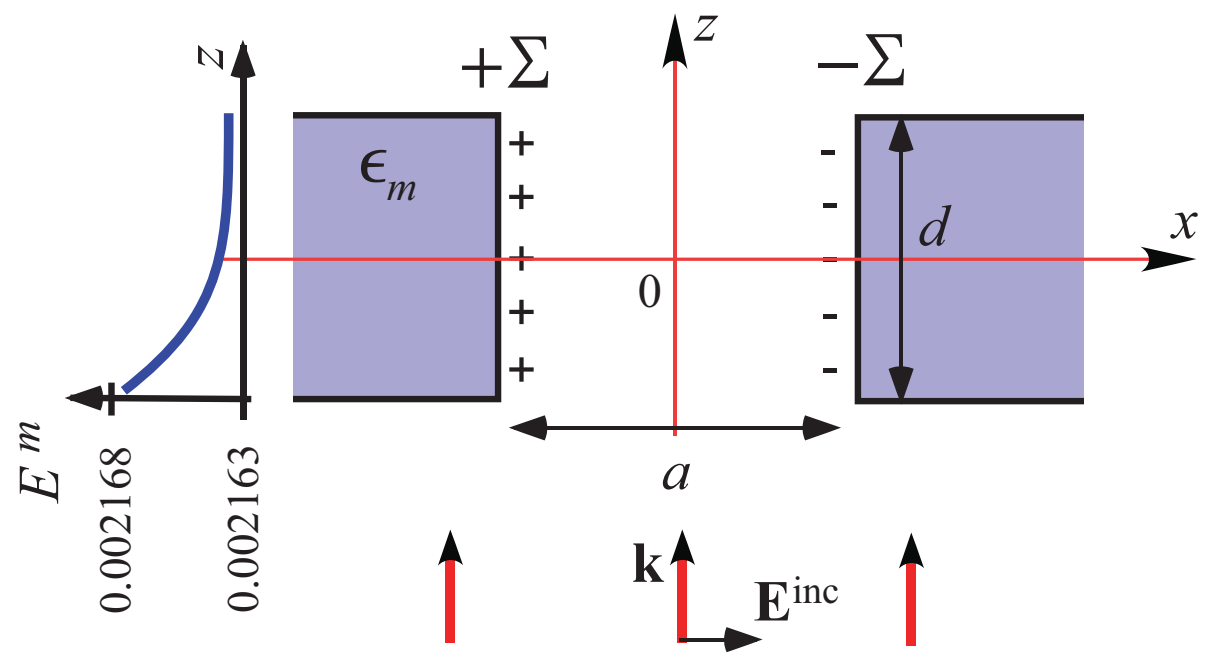

Figure 1: Nanoslit in the metal slab. On the left of the figure the dependence of the electric field inside metal $E^{m}(z)$ is shown (frequency $\omega=2 \pi \times 10^{11} \mathrm{rad} / \mathrm{s}$, amplitude of the incident electric field $\left.E^{i n c}=1\right)$.

is discussed therein, too. Section 4 is devoted to the estimation of the changes introduced by slit shape variations. The fifth section concludes the paper.

\section{Electric field enhancement}

\subsection{Description of the structure}

Let a uniform metal slab of the thickness $d$ less than the typical skin depth on $\mathrm{THz}$ frequencies $\delta$ has a nanoslit of the width $a$ (Figure 1). The slit is illuminated by a monochromatic incident $\mathrm{THz}$ wave, which propagates in the $z$-direction and possesses $x$-polarization. The $x$ - and $y$-sizes of the metal layer are assumed to be infinitely long. The complex dielectric permittivity of the metal is described by the conventional Drude formula

$$
\varepsilon_{m}(\omega)=1-\frac{\omega_{p}^{2}}{\omega(\omega+\mathrm{i} \gamma)},
$$

where $\omega_{p}$ and $\gamma$ are the plasma and decay frequencies, respectively. In the following we consider gold with parameters $\omega_{p}=1.37 \times 10^{4} \mathrm{THz}$ and $\gamma=40.7$ THz taken from Ref. [1].

The unperturbed electric field in the metal slab far from the slit $\mathbf{E}^{m}$ obeys the wave equation and boundary conditions at the metal-air interfaces 
$z= \pm d / 2$. It can be represented in the form

$$
\mathbf{E}^{m}(z)=\left(A_{1} \mathrm{e}^{\eta z}+A_{2} \mathrm{e}^{-\eta z}\right) \mathbf{e}_{x}
$$

where $\eta=\mathrm{i} \sqrt{\varepsilon_{m}} \frac{\omega}{c}$. For good conductors (as it can be attributed to gold at the $\mathrm{THz}$ frequencies), the dielectric permittivity equals $\varepsilon_{m} \approx \mathrm{i} 4 \pi \sigma / \omega$, while parameter $\eta$ is inversely proportional to the skin depth as $\eta=(1-\mathrm{i}) / \delta$. The skin depth is defined in a standard way: $\delta=c / \sqrt{2 \pi \sigma \omega}$, where $c$ is the speed of light in vacuum and $\sigma=\omega_{p}^{2} /(4 \pi \gamma)$ is the metal conductivity. For example, the skin depth $\delta=250 \mathrm{~nm}$ for gold at $\omega=2 \pi \times 10^{11} \mathrm{rad} / \mathrm{s}$ puts an upper constrain on a possible film thickness. Constant coefficients $A_{1}$ and $A_{2}$ can be found from the gold-air boundary conditions. Electric field $E^{m}$ inside the homogeneous sub-skin-depth metal slab with thickness $d=60 \mathrm{~nm}$ is found to be varying very slowly with $z$ (see distribution at the left-hand side in Figure 1). In the following we consider it as independent from $z$.

\subsection{Electric field in the nanoslit}

We arrange two edges of the slit in symmetrical positions at $x=+a / 2$ and $x=-a / 2$ (Figure 1). Electrons affected by the $x$-polarized electric field shift either towards the slit or away from the slit depending on the edge. That is why when at one side of the slit the positive charge is generated, at another side the similar negative charge appears. For the sake of simplicity we assume the charges being at the slit edges only.

The induced charges generate electric field $\mathbf{E}^{s l}$ in the slit. The slit is considerably smaller than the wavelength of the impinging wave $(\sim 3 \mathrm{~mm})$, correspondingly field inside the slit can be approximated as the Coulomb field of charged plates positioned at $x= \pm a / 2$. The $y$-infinite edge surface can be regarded as an infinite homogeneously charged strip. Then the electric field is a superposition of the fields induced by the surface charge densities $\pm \Sigma$ at the gap edges $x= \pm a / 2$. Field $\mathbf{E}^{s l}$ is mainly $x$-polarized. In fact, the boundary conditions at the interfaces $x= \pm a / 2$ provide continuity of the tangential components $\left(E_{y}\right.$ and $\left.E_{z}\right)$. These are small because of the electric field in the metal is small. The normal component of the electric field in the slit $E_{x}^{s l}$ is essential and specified by continuity of the normal component of the displacement vector: $E_{x}^{s l}=\varepsilon_{m} E^{m}$. Even this simple equation shows the existence of the field enhancement effect. As it is seen from Figure 1, for incident field $E^{i n c}=1$ at frequency $\omega=2 \pi \times 10^{11} \mathrm{rad} / \mathrm{s}$ the field in the metal is $E^{m} \approx 0.002$. The dielectric permittivity of metal is $\left|\varepsilon_{m}\right| \approx 7 \times 10^{6}$. So, 
the field enhancement in the very narrow nanoslit, where the homogeneous field inside the slit can be regarded as the field near the boundary, is roughly estimated as $\left(\left|E_{x}^{s l}\right|+E^{i n c}\right) / E^{i n c} \approx 14 \times 10^{3}$. This overestimated value will be corrected below.

Taking constant electric field inside the metal slab, $E^{m}(z) \approx E^{m}(0)$, we suppose that a slit wall can be represented as a $y$-infinite strip with homogeneously distributed charge. Electric field generated by the strip positioned at $x=-a / 2$ and charged with constant surface density $+\Sigma$ equals

$$
E_{x}^{(1)}(x, z)=2 \Sigma\left[\arctan \left(z_{+} / x_{+}\right)+\arctan \left(z_{-} / x_{+}\right)\right],
$$

where $z_{ \pm}=d / 2 \pm z$ and $x_{ \pm}=x \pm a / 2$. The similar formula is applied to the second edge positioned at $x=a / 2$ and charged with $-\Sigma$ :

$$
E_{x}^{(2)}(x, z)=-2 \Sigma\left[\arctan \left(z_{+} / x_{-}\right)+\arctan \left(z_{-} / x_{-}\right)\right] .
$$

The total field generated by the induced charges in the slit is the superposition of the fields defined above:

$$
E_{x}^{s l}(x, z)=E_{x}^{(1)}(x, z)+E_{x}^{(2)}(x, z) .
$$

Eq. (3) is reduced to the well-known field of the infinite homogeneously charged plate $E_{x}=2 \pi \Sigma$, when we approach to the strip, $x_{+} \rightarrow 0$. Arctangent dependence is important for revealing the correct dependencies on the slit sizes.

\subsection{Drude-Lorentz theory of the electron in metal film}

The surface charge density $\Sigma$ can be estimated from the microscopic Lorentz theory as $\Sigma=e N \xi$, where $e$ is the electron charge, $N$ - the freeelectron density in metal, $\xi$ - the electron displacement from the equilibrium position. The charge density $\Sigma$ creates the backward force acting on the electrons as the force that causes plasma oscillations. It can be presented in the form of the average force

$$
\tilde{F}=\frac{1}{l} \int_{-l-a / 2}^{-a / 2} e E(x) d x,
$$

where $E(x)$ is the electric field of the strip like $(3), l$ is the length of the film in $x$ direction. Since $l$ is infinite, we can neglect the contribution of force $\tilde{F}$. 
Nevertheless field gradient induces the non-homogeneous volume charge $\rho(x)=\operatorname{div} \mathbf{E} /(4 \pi)$ in the metal film. This non-constant charge will slow down electrons creating the additional friction force. In average, the force can be estimated as that created by the infinite surface charged with cumulative electric charge $\Sigma^{\prime}=\int_{-\infty}^{-a / 2} \rho(x) d x$ :

$$
F=2 \pi e \Sigma^{\prime}
$$

In principle, the screening due to the medium can be also introduced using the division by the dielectric permittivity. However the screening is not important for the model, because the phenomenological parameter is used further. Using the Coulomb law $d E_{x} / d x=4 \pi \rho$, we have

$$
F=\frac{e}{2}\left(E_{x}^{(1)}(-a / 2,0)+E_{x}^{(2)}(-a / 2,0)\right) .
$$

Friction force $F$ is proportional to the velocity of electrons $\dot{\xi}$, therefore, we choose $\Sigma=e N \dot{\xi} \tau$ in Eqs. (3) and (4), where $\tau$ is a time parameter. It should be noted that $e N \dot{\xi} \tau$ is not the charge at the slit wall, but just an effective charge due to the distribution $\rho(x)$. Finally we get to

$$
F=-m \Gamma \dot{\xi}
$$

where $\Gamma=\left(e^{2} N \tau / 2 m\right)\left[2 \pi-\arctan \left(\frac{d}{2 a}\right)\right]$ is a decay frequency of the additional friction force. Then electron's equation of motion

$$
m(\ddot{\xi}+\gamma \dot{\xi})=e E^{m}-m \Gamma \dot{\xi}
$$

can be rewritten as

$$
\ddot{\xi}+\tilde{\gamma} \dot{\xi}=(e / m) E^{m},
$$

where effective decay frequency $\tilde{\gamma}=\gamma+\Gamma$.

The single unknown parameter can be calibrated from experimental data. Let us assign $\Gamma=\gamma_{0}$ for some gap sizes $a=a_{0}$ and $d=d_{0}$. Then decay frequency $\Gamma$ for the slit with arbitrary dimensions $a$ and $d$ can be expressed in terms of the constant $\gamma_{0}$ as

$$
\Gamma=\gamma_{0} \frac{2 \pi-4 \arctan (d / 2 a)}{2 \pi-4 \arctan \left(d_{0} / 2 a_{0}\right)} .
$$

In our model parameter $\gamma_{0}$ depends only on the metal used. 
Since the fields are monochromatic $\left(E^{m} \sim \exp (-\mathrm{i} \omega t)\right)$ we look for the trial solution of equation (11) in the form $\xi \sim \exp (-\mathrm{i} \omega t)$ and derive

$$
\xi=-\frac{1}{4 \pi e N} \frac{\omega_{p}^{2}}{\omega^{2}+\mathrm{i} \tilde{\gamma} \omega} E^{m}
$$

The charge density at the edge of the gap equals

$$
\Sigma(\omega)=e N \xi=-\frac{1}{4 \pi} \frac{\omega_{p}^{2}}{\omega^{2}+\mathrm{i} \tilde{\gamma} \omega} E^{m} \equiv \frac{1}{4 \pi}\left(\tilde{\varepsilon}_{m}-1\right) E^{m} .
$$

\subsection{Field enhancement}

Knowing the surface charge density (14) electric fields of the strips (3) and (4) can be calculated. Then the averaged electric field in the slit is equal to

$$
\left\langle E_{x}^{s l}\right\rangle=\frac{1}{a d} \int_{-a / 2}^{a / 2} \int_{-d / 2}^{d / 2} E_{x}^{s l}(x, z) d x d z .
$$

We define the field enhancement as $G=\left(\left|\left\langle E_{x}^{s l}\right\rangle\right|+E^{i n c}\right) / E^{i n c}$ accordingly to the definition adopted in [1] (see modelling part). It can be simplified to $G \approx$ $\left|\left\langle E_{x}^{s l}\right\rangle\right|$ taking $E^{i n c}=1$ and assuming $\left|\left\langle E_{x}^{s l}\right\rangle\right| \gg E^{i n c}$. For good conductors at low frequencies $(\omega \ll \gamma<\tilde{\gamma})$ the charge density $\Sigma$ can be accurately approximated by expression $\frac{\mathrm{i} \omega_{p}^{2}}{4 \pi \tilde{\gamma} \omega} E^{m}$. Therefore, the field enhancement in the slit is proportional to $1 / \omega$.

\section{Choice of model parameter and model validation}

\subsection{Model parameter}

First we take the slit with $a=a_{0}=70 \mathrm{~nm}, d=d_{0}=60 \mathrm{~nm}$ to calibrate parameter $\Gamma$ via matching modeling data with the experiment [1]. Employing the single reference point from Ref.[1] we calculate phenomenological parameter $\gamma_{0}=260 \mathrm{THz}$. In our case the reference point corresponds to the enhancement factor $G=1000$ for the frequency $f=0.1 \mathrm{THz}$ (Figure 2(a)). Then other points for plotting the whole curve are obtained with the known frequency dependence of the charge density $\Sigma$. Parameters $\Gamma$ for any other screen's sizes can be calculated from equation (12), e.g., $\Gamma=130 \mathrm{THz}$ for $a=20 \mathrm{~nm}$ and $d=60 \mathrm{~nm}$.

Effective decay frequency $\tilde{\gamma}$ ones calculated can be applied to draw the similar curves for other slit sizes (see Figure 2(b)). All the curves following 

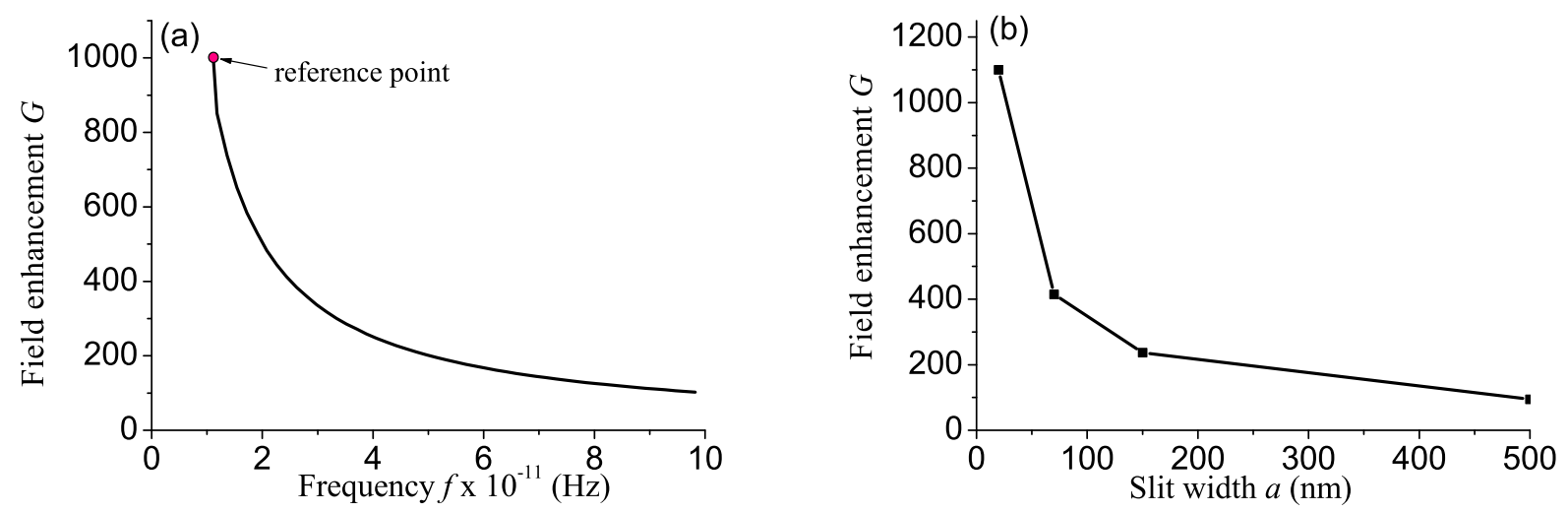

Figure 2: (a) Enhancement $G$ of the electric field in the slit versus frequency for $a=70$ $\mathrm{nm}$ and $d=60 \mathrm{~nm}$. Reference point serves for finding the single parameter of the model. (b) Dependence of the enhancement factor on the slit width $a$ at frequency $f=0.3 \mathrm{THz}$ and $d=60 \mathrm{~nm}$ (the points are chosen to compare with the data in Ref. [1]). We use $\gamma_{0}=260 \mathrm{THz}$ for $a_{0}=70 \mathrm{~nm}, d_{0}=60 \mathrm{~nm}$, and $\omega=2 \pi \times 10^{11} \mathrm{rad} / \mathrm{s}$.

the $1 / \omega$ frequency dependence exhibit quantitative correspondence with the curves reported in Ref. [1].

In Figure 3 the electric field distribution in the $x-z$ cross-section of slits is shown. In general, field is strongly enhanced near the slit edges and reduces to the center of the gap. Field is mainly homogeneous for narrow gaps, when $a \ll d$, as in Figure 3(a). In the other limit of the very broad slit $a \gg d$, field stays intensive near the metal edges and significantly drops near the center of the slit (Figure 3(c)). For the slits of approximately square shapes field maps resemble each other, differing in strength (Figures 3(b) and 3(d)).

The capacitor model $[2,9]$ can be applied for the narrow slits, whose dimensions are defined as $a<d \ll \lambda$, where $\lambda$ is the wavelength. Electric field in the slit can be accurately considered as generated by the surface charges $\Sigma_{0}$ at the gap edges. This field is mostly homogeneous and can be regarded as the field of a capacitor. Then the electric surface charge is equal to

$$
\Sigma_{0}=\frac{c E^{i n c}}{\mathrm{i} \omega 2 \pi d} \approx \frac{7.96 \times 10^{14}}{\mathrm{i} \omega} .
$$

According to our microscopic model the similar surface density (14) takes 

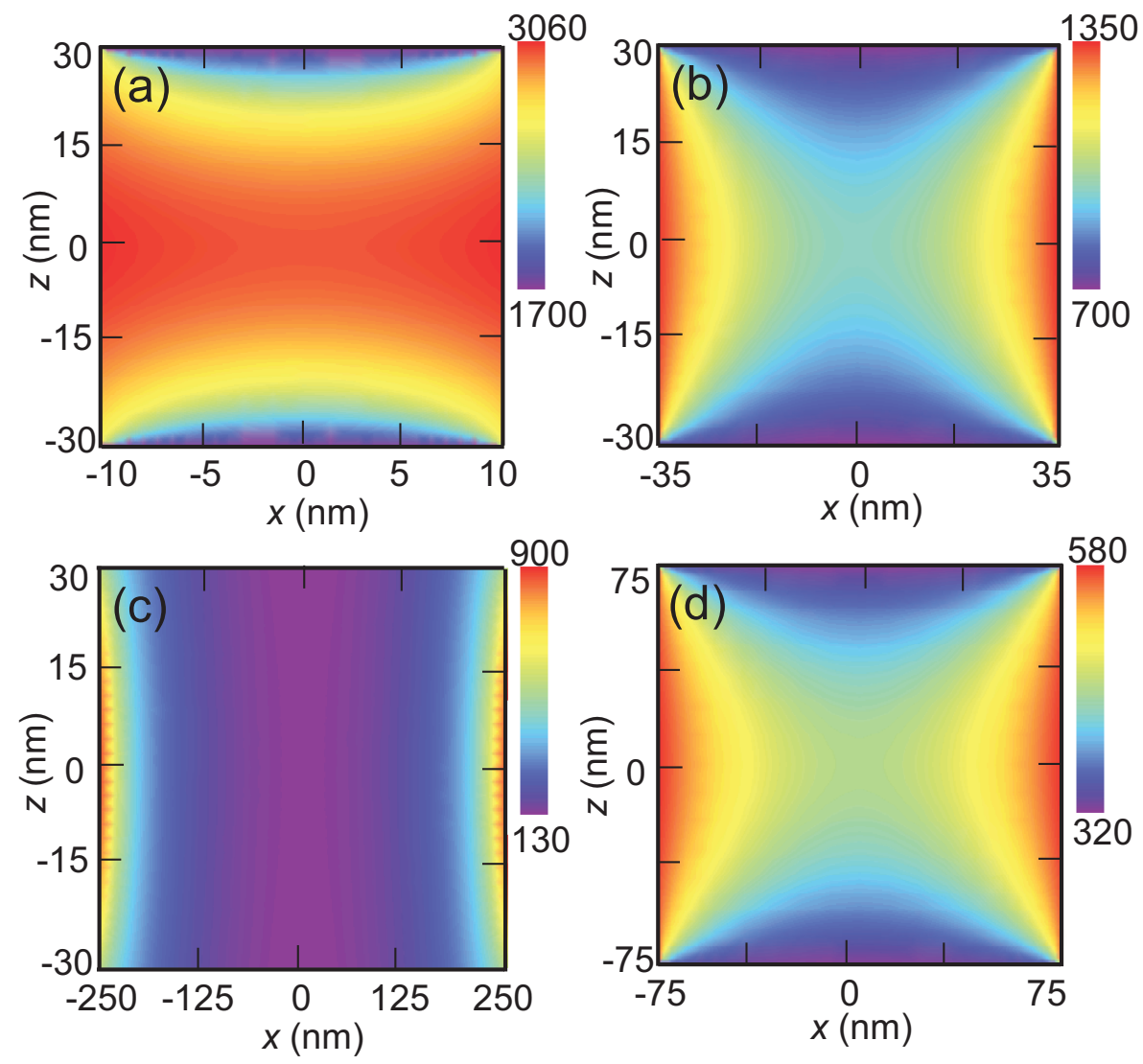

Figure 3: Normalized electric field $\left|E_{x}^{s l}\right|$ inside the slit for (a) $a=20 \mathrm{~nm}, d=60 \mathrm{~nm}$; (b) $a=70 \mathrm{~nm}, d=60 \mathrm{~nm}$; (c) $a=500 \mathrm{~nm}, d=60 \mathrm{~nm} ;$ (d) $a=150 \mathrm{~nm}, d=150$ nm. Parameters: $\gamma_{0}=260 \mathrm{THz}$ for $a_{0}=70 \mathrm{~nm}, d_{0}=60 \mathrm{~nm}$, and $\omega=2 \pi \times 10^{11} \mathrm{rad} / \mathrm{s}$; $E^{i n c}=1$.

the form

$$
\Sigma=-\frac{\omega_{p}^{2} E^{m}}{\mathrm{i} \omega 4 \pi \gamma} \approx-\frac{7.93 \times 10^{14}}{\mathrm{i} \omega}
$$

where $E^{i n c}=1, E^{m}=0.00216, d=60 \mathrm{~nm}$. So, the parametric model produces results, which agree with those from the capacitor model [2, 9]. Note that metal thickness $d$ is accounted in expression (17) through the calculations of electric field in metal $E_{m}$ with the help of the boundary conditions. That is why we have no limitations in our model connected with the case $d \rightarrow 0$ like in equation (16). We use $\gamma$ in equation (17) neglecting $\Gamma$, which is comparatively small for two closely placed charged plates, like the electric 

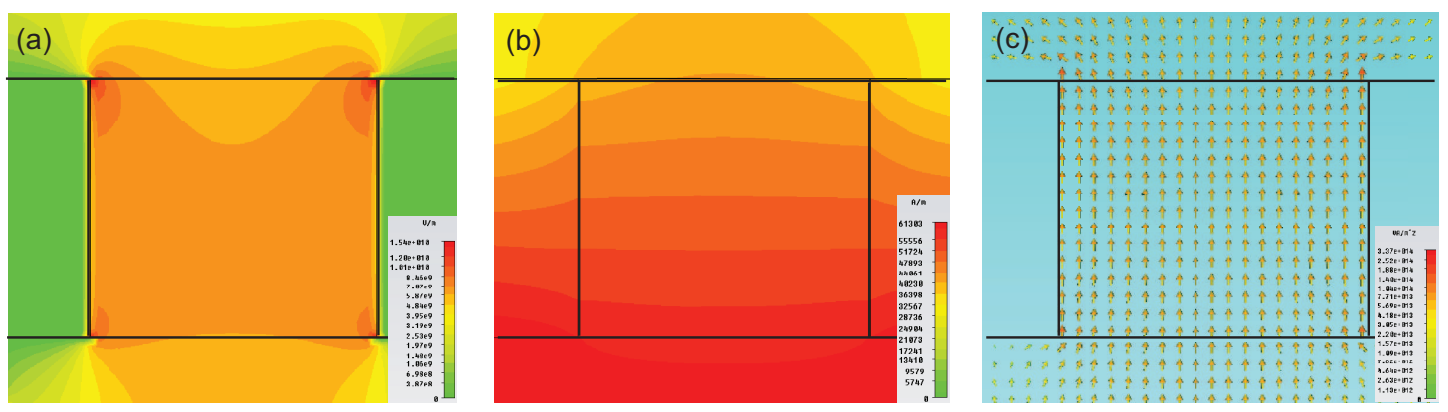

Figure 4: Simulation of the field enhancement phenomenon at $\omega=2 \pi \times 10^{12} \mathrm{rad} / \mathrm{s}$ for the slit with sizes $a=70 \mathrm{~nm}$ and $d=60 \mathrm{~nm}$ : (a) electric field $E_{x}$, (b) magnetic field $H_{y}$, (c) Poynting vector. CST Microwave Studio is used for simulation.

field outside a plane capacitor. When condition $a \ll d$ breaks, expression (16) or our analogue (17) is not more valid. Then one should use the more general formula (14) applicable for any reasonable pairs of $a$ and $d$ quantities.

Another possibility to determine the phenomenological parameter of the model $\gamma_{0}$ is to extract it from simulations. We performed simulation of the fields in the slot at the frequency $\omega=2 \pi \times 10^{12} \mathrm{rad} / \mathrm{s}$ and demonstrate $x$-component of the electric field, magnetic field $H_{y}$ and Poynting vector in Fig. 4.

\subsection{Model features}

In Fig. 4 we observe two important peculiarities of the $E_{x}$ field distribution. First, there is the field increase in vicinity of the metal corners. Second, the picture is asymmetric along $z$ axis, so that $E(z) \neq E(-z)$. These two features are clearly visible in Fig. 5, where we show field distributions along two axies of symmetry. We wish to compare the electric field distributions from simulations and that from the microscopic model. Averaging the electric field in the slit we find the enhancement factor $G=247$ which corresponds to $\gamma_{0}=81 \mathrm{THz}$ providing the matching enhancement $G$.

Using the model parameter found from the simulation we can now calculate the electric field distribution according to our model. In general, the distribution is not similar to that in simulation - it is symmetric and have no corner effects (see Fig. 3). Nevertheless, in average the field behaves in the correct way: along $z$, it is greatest at the center $x=0, z=0$; along $x$, it is smallest at the center. In spite of the local fields are substantially different along the line $z=0$, this difference is essentially mitigated by averaging of 

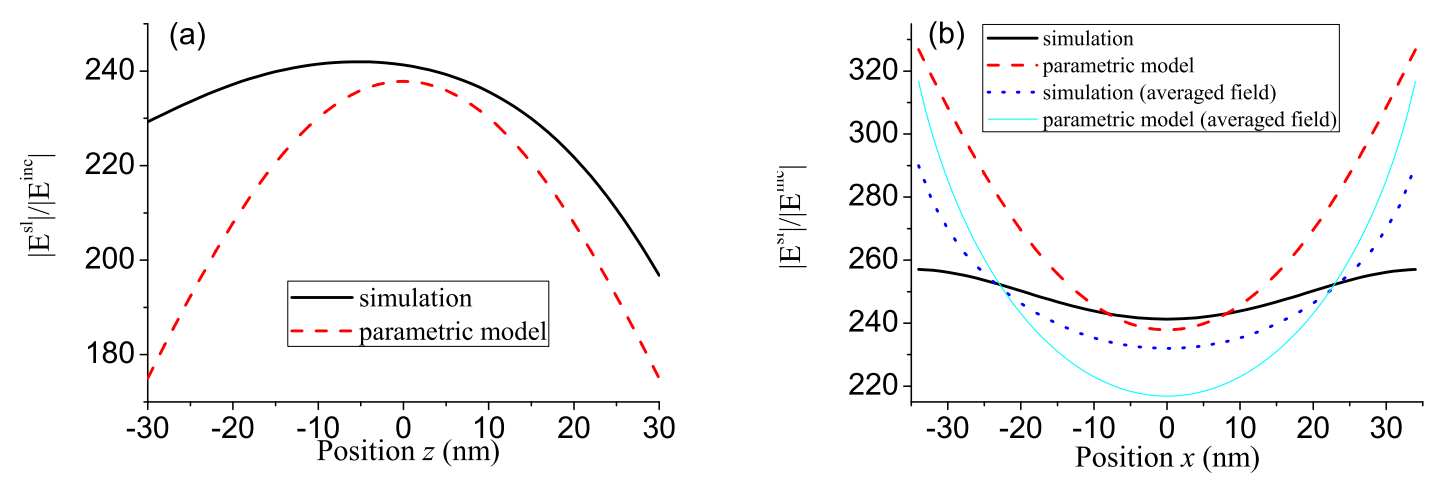

Figure 5: Comparison of the electric fields $E_{x}$ of simulation and parametric model: (a) $z$-dependence at $x=0$, (b) $x$-dependence at $z=0$. Parameters: $\gamma_{0}=81 \mathrm{THz}$ for $a_{0}=70$ $\mathrm{nm}, d_{0}=60 \mathrm{~nm}$, and $\omega=2 \pi \times 10^{12} \mathrm{rad} / \mathrm{s}$.

the electric field $(1 / d) \int_{-d / 2}^{d / 2} E_{x}(x, z) d z$ (Fig. $\left.5(\mathrm{~b})\right)$. Then the asymmetry effects disappear and values from our model and simulations differ approximately by $10 \%$. It should be noted that the deviations from the parametric model indicate the existence of the corner and wave effects, which can be neglected, if the accurate calculations are not necessary.

Using our model we can predict the enhancement factors for different metals. We need two things for that: Drude parameters of the material and the single value of the enhancement factor for some geometrical parameters of the slit. Fortunately, the enhancement factors $G$ coincide for the ordinary metals. It can be understood from the similarity of the metal to the perfect electric conductor within $\mathrm{THz}$ frequency range, when the dielectric permittivity is very large. This assertion is confirmed by the numerical calculations (not shown). So, we can use $G=1000$ for the geometrical parameters $a=70$ $\mathrm{nm}$ and $d=60 \mathrm{~nm}$ and frequency $\omega=2 \pi \times 10^{11} \mathrm{rad} / \mathrm{s}$. Using the Drude parameters of the metals [17] we find parameter $\gamma_{0}$ for them (see Table 1). From the table we can notice that $\gamma_{0} \approx 6.4 \gamma$. In this way we can use metalindependent parameter (equal 6.4 in our case) instead of the decay frequency parameter $\gamma_{0}$.

The model has two major limitations. First, the thickness of the metal film $d$ cannot be large, because we use the assumption that electric field in the metal is homogeneous, i.e. $E^{m}=E^{m}(z=0)$. If $d$ is greater than several skin depths $\delta$, the model should be extended. Second, the model is not valid 
Table 1: Parameters $\gamma_{0}$ for different metal parameters given in [17].

\begin{tabular}{cccc}
\hline \hline Metal & $\gamma(\mathrm{THz})$ & $\omega_{p}(\mathrm{THz})$ & $\gamma_{0}(\mathrm{THz})$ \\
\hline $\mathrm{Al}$ & 122 & $2.24 \times 10^{4}$ & 781 \\
$\mathrm{Cu}$ & 52.4 & $1.2 \times 10^{4}$ & 335 \\
$\mathrm{Au}$ & 40.7 & $1.37 \times 10^{4}$ & 260 \\
$\mathrm{~Pb}$ & 27.3 & $1.17 \times 10^{4}$ & 175 \\
$\mathrm{Ag}$ & 27.3 & $1.37 \times 10^{4}$ & 175 \\
$\mathrm{~W}$ & 81.6 & $0.91 \times 10^{4}$ & 518 \\
\hline
\end{tabular}

for the metal films of finite size in $x$ direction. In this case the electron oscillations in the sample can play important part and the field enhancement substantially reduces.

\section{Slit shape effect}

Our model can easily incorporate the finite sizes of the slits in the $y$ direction. To observe the qualitative picture we replace a strip with a thread placed at $z=0$ and linearly charged with density $\kappa$. This approximation is valid for not very short strip lengths $b$ in the $y$-direction $(b>>a, d)$. The accurate calculation of strips field is expected to result in the similar $b$ dependence of the enhancement, because the strip resembles thread for large $b$. The thread creates the following electric field at the distance $x$ from it:

$$
E^{t h r}(x, b)=2 \kappa \arctan (b / 2 x) .
$$

Field in the gap $\mathbf{E}_{x}^{\prime s l}(x, z)=p \mathbf{E}_{x}^{s l}(x, z)$ is changed by $p$ times compared with the case of an infinite strip (thread), thus bringing the enhancement factor to $p G_{0}$, where $p$ is

$$
p=\frac{E^{t h r}(a / 2, b)}{E^{t h r}(a / 2, \infty)}=\frac{2 \arctan (b / a)}{\pi} .
$$

However, the screening field has to be adjusted as well. Field generated by one strip at the center of another strip is changed in $w=E^{t h r}(a, b) / E^{t h r}(a, \infty)=$ $2 \arctan (b / 2 a) / \pi$ times. So, according to (3) and (4) fields at the edges are $\mathbf{E}_{x}^{\prime(1)}(a / 2, z)=w \mathbf{E}_{x}^{(1)}(a / 2, z)$ and $\mathbf{E}_{x}^{\prime(2)}(-a / 2, z)=w \mathbf{E}_{x}^{(2)}(-a / 2, z)$. Such change affects the decay frequency, which now takes the form

$$
\gamma^{s c r}(w)=\gamma_{0} \frac{2 \pi-4 w \arctan (d / 2 a)}{2 \pi-4 \arctan \left(d_{0} / 2 a_{0}\right)} .
$$


Eventually, the new enhancement factor $G$ can be approximately expressed via the enhancement factor of the infinite strip $G_{0}$ as follows

$$
G \approx \frac{\gamma^{s c r}+\gamma}{\gamma^{s c r}(w)+\gamma} p G_{0}
$$

Expectedly the field enhancement factor is small for short strips irrespectively that our thread model fails. It significantly increases when $b$ extends from $10 \mathrm{~nm}$ to $1000 \mathrm{~nm}$ (see Figure 6). For the lengthy slit dimensions in $y$ direction $(b>1 \mu \mathrm{m})$, the field enhancement is near $G_{0}$.

Our model can be extended in principle on slits with shapes different from rectangular. In spite of the fact that the shape significantly influences the field distribution inside the gap, it does not play an important role for the enhancement $G$, which is kind of integral characteristics, see Eq.(15). Since field $E^{s l}$ is defined as the superposition of the Coulomb fields created by the charges at the interfaces of the slit, the enhancement factor mainly depends on the area of the slit interfaces in a simple way: the larger the area, the bigger the gain $G$. Therefore, in estimation of the enhancement factor we can approximate any slit shape by a rectangular slit with effective dimensions $a$ and $b$ along $x$ and $y$ axes, respectively.

\section{Conclusion}

We proposed a one-parametrical model describing the $\mathrm{THz}$ electric field behavior in a nanometer-scaled slit in a thin metal film. Our model was corroborated in testing versus experimental results. It demonstrates complete qualitative relevance and good quantitative agreement with the previously published results [1]. The advantages of the model are its simplicity giving a clear insight in the physics of ongoing processes and presence of only one fitting parameter, easily deducible from the experimental or simulation data. Thus it can be employed to map fields in the zone of considerable enhancement or to make semi-quantitative predictions, for example concerning slits shape or size.

The model can be applied to estimate the violation of the Babinet principle predicted in Ref. [18] for the sub-skin-depth regime. According to Ref. [18] we shall consider the complimentary structures: metallic nanogap and metallic nanowire of the same sizes illuminated by the TM and TE waves, respectively. Electric field enhancement for the nanogap has been discussed above and can be estimated in terms of the electric field at the center of the 


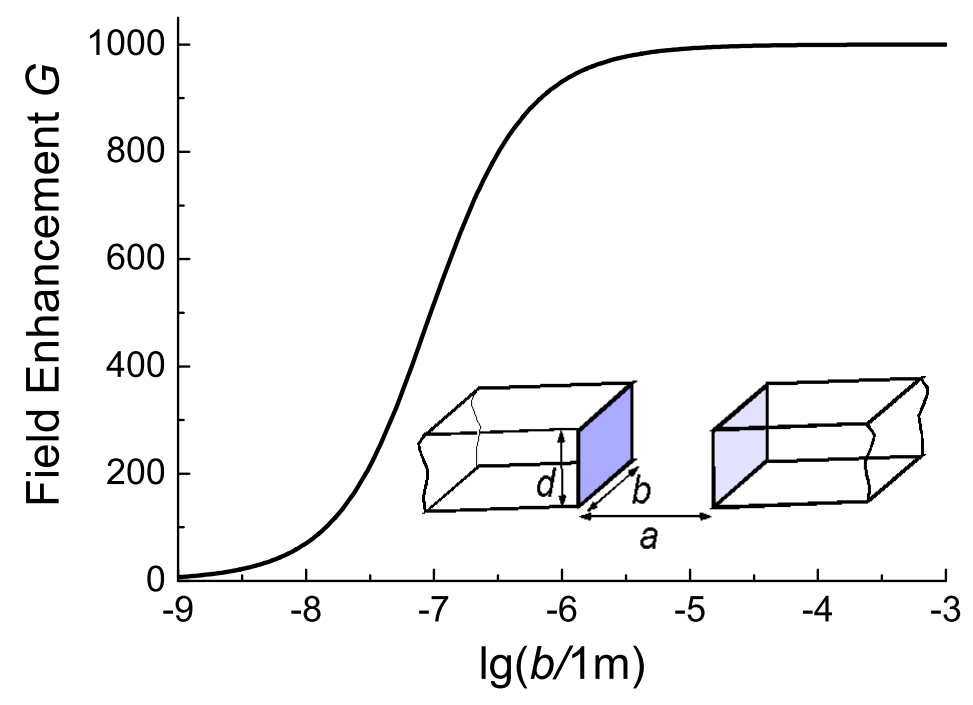

Figure 6: Enhancement $G$ of the electric field in the rectangular slit vs. the gap dimension b. Parameters: $G_{0}=1000, \gamma_{0}=260 \mathrm{THz}, a_{0}=70 \mathrm{~nm}, d_{0}=60 \mathrm{~nm}$.

slit $G_{E}=8 \Sigma \arctan (d / a) / E^{i n c}$. Magnetic field in the nanowire is generated by the electric current created by the incident electric field $E_{y}$. The magnetic field enhancement follows from Ampire's law $G_{H}=2 \pi j a d /\left(c(d+a) H^{i n c}\right)$, where $j$ is the electric current density. Remembering that $E^{i n c}=H^{i n c}$ and neglecting $\omega^{2}$ in comparison with $\gamma \omega$ we derive

$$
\left|\frac{G_{H}}{G_{E}}\right|=\frac{\pi \omega a d \tilde{\gamma}}{4 c(d+a) \arctan (d / a) \gamma}
$$

This formula provides only the rough estimation, but we can see that for the small frequencies (large skin depths) the ratio $\left|G_{H} / G_{E}\right|$ is small (Babinet principle is violated). When increasing frequency the skin depth becomes smaller than the thickness of the gap and wire, while the quantity $\left|G_{H} / G_{E}\right|$ becomes greater and the enhancements tend to be equal (Babinet principle holds true).

Financial support from the Danish Research Council for Technology and Production Sciences via projects THz COW and COST Action MP0702 is acknowledged. Also, we thank Prof. S.A. Tretyakov for the fruitful discussion of the model, Dr. J.-H. Kang, Prof. D.S. Kim, and S.M. Koo for their 
kind support in providing us with detailed data of their published numerical simulations.

\section{References}

[1] Seo M A, Park H R, Koo S M, Park D J, Kang J H, Suwal O K, Choi S S, Planken P C M, Park G S, Park N K, Park Q H and Kim D S 2009 Terahertz field enhancement by a metallic nano slit operating beyond the skin-depth limit Nat. Phot. 3 152-6

[2] Park Q-H 2009 Optical antennas and plasmonics Contemporary Physics $50407-23$

[3] Ebbesen T W, Lezec H J, Chaemi H F, Thio T and Wolff H F 1998 Extraordinary optical transmission through sub-wavelength hole arrays Nature $391667-9$

[4] Garcia-Vidal F J, Martin-Moreno L, Ebbesen T W and Kuipers L 2010 Light passing through subwavelength apertures Rev. Mod. Phys. 82 72987

[5] Park D J, Choi S B, Ahn Y H, Rotermund F, Sohn I B, Kang C, Jeong M S and Kim D S 2009 Terahertz near-field enhancement in narrow rectangular apertures on metal film Opt. Express 17 12495-501

[6] Lee J W, Park T H, Nordlander P and Mittleman D M 2009 Terahertz transmission properties of an individual slit in a thin metallic plate Opt. Express 17 12660-7

[7] Bethe H A 1944 Theory of diffraction by small holes Phys. Rev. 66 163-82

[8] Bouwkamp C J 1954 Diffraction theory Rep. Progr. Phys. XVIII 35-100

[9] Kang J H, Kim D S and Park Q-H 2009 Local Capacitor Model for Plasmonic Electric Field Enhancement Phys. Rev. Lett. 102093906

[10] Zhang X, Li C-F 2009 Polarization-Independent Directional Beaming of Light by a Subwavelength Metal Slit Chin. Phys. Lett. 26114204

[11] Tao J, Huang X G and Liu S H 2010 Optical characteristics of surface plasmon nanonotch structure J. Opt. Soc. Am. B 27 1430-4 
[12] Park H R, Koo S M, Suwal O K, Park Y M, Kyoung J S, Seo M A, Choi S S, Park N K, Kim D S and Ahn K J 2010 Resonance behavior of single ultrathin slot antennas on finite dielectric substrates in terahertz regime Appl. Phys. Lett. 96211109

[13] Chen H T, Padilla W J, Zide J M O, Gossard A C, Taylor A J and Averitt R D 2006 Active terahertz metamaterial devices Nature 444 597-600

[14] Chen H T, Padilla W J, Cich M J, Azad A K, Averitt R D and Taylor A J 2009 A metamaterial solid-state terahertz phase modulator Nat. Photonics 3 148-51

[15] Dai D and He S 2009 A silicon-based hybrid plasmonic waveguide with a metal cap for a nano-scale light confinement Opt. Express 17 16646-53

[16] Oulton R F, Sorger V J, Genov D A, Pile D F P and Zhang X 2008 A hybrid plasmonic waveguide for subwavelength confinement and longrange propagation Nat. Photonics 2 496-500

[17] Ordal M A, Long L L, Bell R J, Bell S E, Alexander Jr R W and Ward C A 1983 Optical properties of the metals Al, Co, Cu, Au, Fe, Pb, Ni, $\mathrm{Pd}, \mathrm{Pt}, \mathrm{Ag}, \mathrm{Ti}$, and $\mathrm{W}$ in the indrared and far infrared Appl. Opt. 22 1099-120

[18] Koo S, Kumar M S, Shin J, Kim D S, and Park N 2009 Extraordinary Magnetic Field Enhancement with Metallic Nanowire: Role of Surface Impedance in Babinets Principle for Sub-Skin-Depth Regime Phys. Rev. Lett. 103263901 